\title{
Dark-State Cooling of Atoms by Superfluid Immersion
}

\author{
A. Griessner, ${ }^{1,2}$ A. J. Daley, ${ }^{1,2}$ S. R. Clark, ${ }^{3}$ D. Jaksch, ${ }^{3}$ and P. Zoller ${ }^{1,2}$ \\ ${ }^{1}$ Institute for Quantum Optics and Quantum Information of the Austrian Academy of Sciences, A-6020 Innsbruck, Austria \\ ${ }^{2}$ Institute for Theoretical Physics, University of Innsbruck, A-6020 Innsbruck, Austria \\ ${ }^{3}$ Clarendon Laboratory, University of Oxford, Parks Road, Oxford OX1 3PU, United Kingdom
}

(Received 10 July 2006; published 29 November 2006)

\begin{abstract}
We propose and analyze a scheme to cool atoms in an optical lattice to ultralow temperatures within a Bloch band and away from commensurate filling. The protocol is inspired by ideas from dark-state laser cooling but replaces electronic states with motional levels and spontaneous emission of photons by emission of phonons into a Bose-Einstein condensate, in which the lattice is immersed. In our model, achievable temperatures correspond to a small fraction of the Bloch bandwidth and are much lower than the reservoir temperature. This is also a novel realization of an open quantum optical system, where known tools are combined with new ideas involving cooling via a reservoir.
\end{abstract}

DOI: 10.1103/PhysRevLett.97.220403

PACS numbers: 03.75.Lm, 32.80.Pj, 42.50.- $\mathrm{p}$

Fundamental advances in atomic physics are often linked to the development of novel cooling methods, as illustrated by laser and evaporative cooling, which led to the recent realization of degenerate Bose and Fermi gases [1]. This has further led to the achievement of strongly correlated atomic ensembles in the lowest Bloch band of an optical lattice [2-5]. However, in order to realize some of the most interesting condensed matter phases predicted for lattice Hamiltonians, even better purification of the motional state is necessary, in particular, for atoms in a partially filled Bloch band [5]. Here we propose a method for cooling atoms to mean energies much smaller than the width of the lowest Bloch band $4 J^{0}$. In our setup [cf. Fig. 1(a)], lattice atoms $a$ are excited to the first Bloch band via a Raman laser pulse except when they occupy Bloch states with quasimomentum close to zero-socalled dark states. The lattice is immersed in a BoseEinstein condensate (BEC) of a different atomic species $b$, so that the atoms can subsequently decay back to the lowest band via collisional interactions with the BEC reservoir [6,7]. Thus, the atom is recycled to the lowest band by emission of a phonon-or more precisely, a Bogoliubov excitation [6]. By repeated application of laser excitation and "spontaneous emission," cooling into the dark-state region of quasimomenta is achieved without loss of atoms [8] [cf. Fig. 1(b)].

This method is inspired by the seminal Kasevich-Chu scheme [11] for subrecoil laser cooling [12,13] but replaces internal atomic states by Bloch band excitations and spontaneously emitted photons by phonons. Our scheme thus operates on a much smaller energy scale than laser cooling, with correspondingly lower temperatures. This method can also be seen as a form of sympathetic cooling, where the energy is removed by phonons with energies equal to the Bloch band separation. Such phonon modes will initially be in the vacuum state, giving an effective $T=0$ reservoir, and allowing temperatures significantly lower than the BEC reservoir temperature, in contrast to standard sympathetic cooling. The ability to switch the collisional interactions via Feshbach resonances [14] enables us to study the cooling scenario in the weakly interacting gas, creating strongly correlated phases by ramping up the interaction in a final step.

On a formal level, our cooling scheme can be written as the iterative application of a map:

$$
\mathcal{M}_{j}: \hat{\rho}_{j} \rightarrow \hat{\rho}_{j+1} \equiv\left(\hat{\mathcal{D}} \circ \hat{E}_{j}\right) \hat{\rho}_{j},
$$

where the density operator $\hat{\rho}_{j}$ describes the atoms in the lowest Bloch band before the $j$ th step. Each step consists of two parts: the coherent laser excitation $\hat{E}_{j}$ of lattice atoms $a$ and the dissipative decay $\hat{D}$ returning atoms to the lowest band via coupling to the reservoir $b$ (Fig. 1). To achieve the best possible cooling, differently shaped excitation pulses $\hat{E}_{j}, j=0, \ldots, N_{p}-1$, are applied, and this sequence is repeated, with $\hat{E}_{j}=\hat{E}_{j \bmod N_{p}}$. This repeated application of the map corresponds to a purification of the density opera-
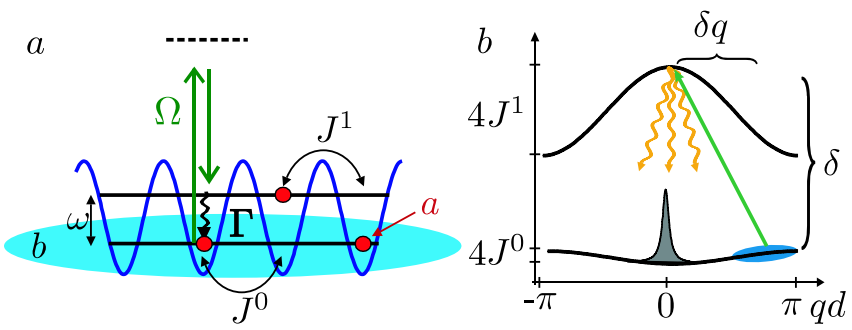

FIG. 1 (color online). (a) Cooling setup: Atoms $a$ in an optical lattice are coupled to the first excited motional state via a Raman process and decay to the ground motional state due to collisional interactions with a BEC of species $b$ in which the lattice is immersed. Tunneling between neighboring sites with amplitude $J^{\alpha}$ gives rise to Bloch bands. (b) Momentum space picture: Atoms with higher quasimomentum $q$ are excited to the upper Bloch band and decay to random quasimomentum states. After several cycles, atoms $a$ collect in a dark-state region near $q=0$ with low excitation probability. 
tor, starting from an initial mixed state (e.g., a thermal distribution) towards a pure state (at zero temperature $T=$ $0)$. In order to find appropriate forms of the Raman pulses and the action of $\hat{D}$, we analyze the dynamics of the lattice atoms and their interaction with the reservoir gas.

We consider a one-dimensional model for the motion of atoms $a$, which is readily generalized to higher dimensions (with arguments similar to Ref. [11]). Including Raman coupling, the Hamiltonian is $\hat{H}_{a}=\hat{H}_{0}+\hat{H}_{I}$, with

$$
\begin{aligned}
\hat{H}_{0}= & \sum_{q, \alpha} \varepsilon_{q}^{\alpha}\left(\hat{A}_{q}^{\alpha}\right)^{\dagger} \hat{A}_{q}^{\alpha}+(\omega-\delta) \sum_{q}\left(\hat{A}_{q}^{1}\right)^{\dagger} \hat{A}_{q}^{1} \\
& +\frac{\Omega}{2} \sum_{q}\left[\left(\hat{A}_{q}^{1}\right)^{\dagger} \hat{A}_{q-\delta q}^{0}+\text { H.c. }\right], \\
\hat{H}_{I}= & \frac{1}{2} \sum_{i, \alpha} U^{\alpha \alpha} \hat{n}_{i}^{\alpha}\left(\hat{n}_{i}^{\alpha}-1\right)+2 U^{10} \sum_{i} \hat{n}_{i}^{1} \hat{n}_{i}^{0} .
\end{aligned}
$$

Here $\hat{A}_{q}^{\alpha}$ and $\left(\hat{A}_{q}^{\alpha}\right)^{\dagger}$ are annihilation and creation operators, respectively, for quasimomentum $q$ in Bloch band $\alpha \in$ $\{0,1\}$, satisfying Bose or Fermi (anti)commutation relations. The kinetic energy is $\varepsilon_{q}^{\alpha}=-2 J^{\alpha} \cos (q d)$, where $d$ is the lattice spacing, $J^{\alpha}$ are the tunneling amplitudes [with $J^{0}>0$ and $J^{1}<0$; see Fig. 1(b)], and $\omega$ is the band separation. The effective Rabi frequency $\Omega=$ $\Omega_{R} \int d x \exp (-i \delta q x) w^{1}(x) w^{0}(x)$, where $\Omega_{R}$ is the two photon Rabi frequency as a function of time during the pulse and $w^{\alpha}(x)$ the Wannier functions. The Hamiltonian is a two-band model, written in a rotating frame with Raman detuning $\delta$, and $\delta q$ denotes the momentum transfer. We choose units $\hbar=k_{B}=1$, where $k_{B}$ is the Boltzmann constant. The parameters $\Omega, \delta$, and $\delta q$ will change during the pulse sequence but be constant during a given pulse $j$. On site interactions between lattice atoms are represented by $\hat{H}_{I}$, with $\hat{n}_{i}^{\alpha}$ the number operator for atoms in site $i$ and band $\alpha$ and $U^{\alpha \alpha^{\prime}}$ the associated on site energy shifts [2].

The density-density interaction between lattice atoms $a$ and a three-dimensional BEC reservoir $b$, which gives rise to the decay $\hat{\mathcal{D}}$, is described by the Hamiltonian [6]

$$
\hat{H}_{\mathrm{int}}=\sum_{\alpha, \alpha^{\prime}} \sum_{\mathbf{k}, q}\left[G_{\alpha, \alpha^{\prime}}^{\mathbf{k}} \hat{b}_{\mathbf{k}}\left(\hat{A}_{q}^{\alpha}\right)^{\dagger} \hat{A}_{q-k}^{\alpha^{\prime}}+\text { H.c. }\right] \text {. }
$$

Here the operator $\hat{b}_{\mathbf{k}}^{\dagger}$ creates a Bogoliubov excitation with momentum $\mathbf{k}=\left(k, k_{y}, k_{z}\right)$ and, neglecting overlap of Wannier functions in different lattice sites, the coupling $G_{\alpha, \alpha^{\prime}}^{\mathbf{k}} \approx g_{a b}\left(S(\mathbf{k}) \rho_{b} / V\right)^{1 / 2} \int d^{3} x e^{i \mathbf{k} \mathbf{x}} w^{\alpha}(\mathbf{x}) w^{\alpha^{\prime}}(\mathbf{x})$. The strength of the interspecies contact interaction is denoted $g_{a b}, \rho_{b}$ and $V$ are the density and volume, respectively, of the BEC reservoir, and $S(\mathbf{k})$ is the static structure factor [1]. For excitation energies less than the chemical potential $\mu$, excitations are sound waves for which $S(\mathbf{k})$ is strongly suppressed, and $S(\mathbf{k}) \rightarrow 0$ as $|\mathbf{k}| \rightarrow 0$ [1]. For energies larger than $\mu$, excitations are in the particlelike sector of the spectrum, with much larger $S(\mathbf{k}) \rightarrow 1$. Here we will typically have $4 J^{0}<\mu<\omega$, so that decay between bands is induced by particlelike excitations with strong coupling, but collisional processes between the reservoir and atoms in the lowest Bloch band are suppressed. In close analogy to Refs. [6,7], we derive a master equation for the reduced system density operator, describing the decay between bands in the Born-Markov approximation [15]. The associated Liouvillian is $\mathcal{L}[\rho]=\sum_{k} \Gamma_{k}\left(2 c_{k} \rho c_{k}^{\dagger}-c_{k}^{\dagger} c_{k} \rho-\right.$ $\left.\rho c_{k}^{\dagger} c_{k}\right) / 2$. The momentum $k$ along the lattice axis is bounded by $|k| \leq \sqrt{2 m_{b} \omega}$ due to energy conservation, where $m_{b}$ is the mass of atoms $b$, and the jump operators $c_{k}$ are defined as $c_{k}=\sum_{q}\left(A_{q-k}^{0}\right)^{\dagger} A_{q}^{1}$. The resulting decay rates $\Gamma_{k}$ for spontaneous emission of a phonon with momentum $k$ projected on the axis of the lattice can be written explicitly for deep lattices, where $\omega \gg\left|J^{1}\right|, J^{0}$ and the individual lattice sites can be approximated as harmonic oscillator potentials. We find $\Gamma_{k}=$ $g_{a b}^{2} \rho_{b} m_{a} a_{0}^{2} k^{2} \exp \left(-a_{0}^{2} k^{2} / 2\right) / 2 L$, with $a_{0}$ the size of the ground state in each lattice site, $m_{a}$ the mass of atoms $a$, and $L$ the length of the 1D lattice. We denote the total decay rate from the excited band by $\Gamma=\sum_{k} \Gamma_{k}$. We consider a situation where dissipation is switched off during the excitation step, so that $\hat{E}_{j}$ and $\hat{D}$ occur separately. This can be achieved, e.g., by tuning the collisional interaction so that $g_{a b} \approx 0$. We can read the action of $\hat{E}_{j}$ and $\hat{D}$ for a given step $j$ from the master equation.

We first illustrate the cooling process for a single lattice atom, designing a sequence of Raman laser pulses, where the $j$ th pulse excites the atom with initial quasimomentum $q$ in the lowest band to the first excited band with probability $P_{j}(q)$. We require $P_{j}(q)=0$ for $q \approx 0$, but $P_{j}(q) \rightarrow$ 1 for states with high quasimomentum [cf. Fig. 1(b)]. In analogy with Raman cooling schemes in free space [11], we choose square pulses with duration $\tau_{j}=\pi / \Omega_{j}$, for which $P_{j}(q)=\sin ^{2}\left(\sqrt{\delta_{q+\delta q_{j}}^{2}+\Omega_{j}^{2}} \tau_{j} / 2\right) \Omega_{j}^{2} /\left(\delta_{q+\delta q_{j}}^{2}+\right.$ $\Omega_{j}^{2}$, with the effective detuning $\delta_{q+\delta q_{j}} \equiv \omega+\varepsilon_{q+\delta q_{j}}^{1}-$ $\varepsilon_{q}^{0}-\delta_{j}$.

An example of an efficient pulse sequence is shown in Figs. 2(a)-2(c). We begin with an intense laser pulse which resonantly excites atoms with momentum $q \sim \pi / d$ around the edges of the Brillouin zone [Fig. 2(a)]. The subsequent pulses move the resonant transition closer to $q=0$ by adjusting the momentum transfer $\delta q_{j}$ and Raman detuning $\delta_{j}$ [Fig. 2(b)]. In order to prevent excitation of atoms with $q=0$, we decrease $\Omega$ and increase the pulse duration $\tau$ for the later pulses, each time achieving $P_{j}(q=0)=0$. To resolve the transition, we must always have $\Omega \ll 8\left|J^{1}\right|$ and, therefore, $\tau \gg \pi / 8\left|J^{1}\right|$. Note that as the energy of the excited band varies more rapidly with $q$, it is the value of $J^{1}$ and not $J^{0}$ that gives the resolution of the excitation. However, the relationship between $J^{1}$ and $J^{0}$ is fixed by the lattice depth (e.g., for the parameters used in Figs. 2 and $3, V_{0}=10 \omega_{R}$, we have $J^{0}=0.019 \omega_{R}$ and $J^{1}=$ $\left.-0.25 \omega_{R}\right)$. By combining a sequence of 5 pulses, one can efficiently excite most atoms with $|q|>0$, as shown in Fig. 2(c). 


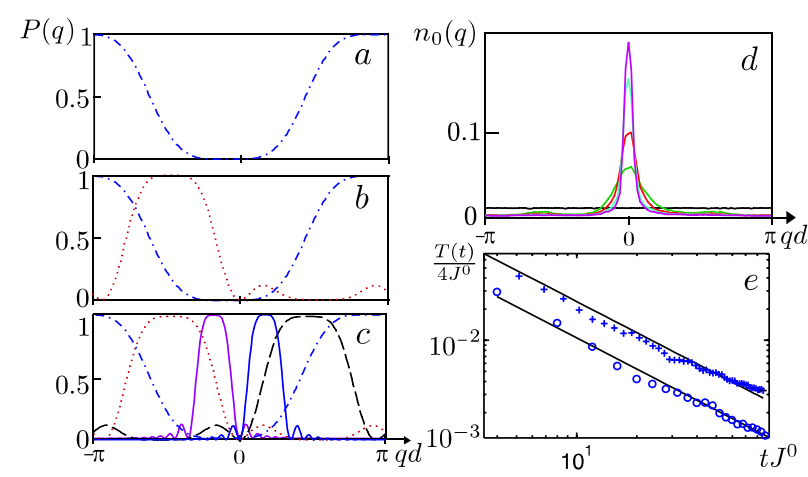

FIG. 2 (color online). (a) - (c) Excitation probability $P_{j}(q)$ for a sequence of $N_{p}=5$ pulses: first [(a)-(c), dashed-dotted line], second [(b),(c), dotted line], and the remaining pulses (c). Parameters used: $\Omega=(27.9,13.7,13.7,2.37,2.37) J^{0}, \quad \delta q=$ $(0.16,-1.75,1.75,-2.63,2.63) / d, \quad$ and $\quad(\delta-\omega)=-(28.4$, $25.8,25.8,24.7,24.7) J^{0}$ for the different pulses. (d) Successive narrowing of the momentum distribution in the lower Bloch band after $0,1,3,5$, and 10 cooling cycles from numerical simulations ( $M=101$ lattice sites) based on the pulse sequence in (a)-(c). We choose parameters for ${ }^{87} \mathrm{Rb}$ in the lattice and ${ }^{23} \mathrm{Na}$ in the reservoir, with $\Gamma=53 J^{0}$ from $m_{a} / m_{b}=3.73, \quad \rho_{b}=$ $5 \times 10^{14} \mathrm{~cm}^{-3}$, and scattering length $a_{a b}=14 \mathrm{~nm}$. $V_{0}=$ $10 \omega_{R}$, and $\omega_{R}=2 \pi \times 3.8 \mathrm{kHz}$. (e) Temperature vs time for a single atom: Crosses and circles denote numerical results and solid lines analytical results based on Lévy statistics. Pulse sequences for circles: Same as in (d); crosses: $N_{p}=3$ pulses with $\Omega=(32.6,7.9,7.9) J^{0}, \quad \delta q d=(0.31,2.12,-2.12)$, and $(\delta-\omega)=-(28.4,25.3,25.3) J^{0}$.

To quantitatively analyze the cooling process, we numerically simulate the time evolution of the density operator $\hat{\rho}$ using quantum trajectories [17] starting from a completely mixed state in the lowest band $\left(T \gg 4 J^{0}\right)$, with $\sim 10^{5}$ trajectories. During the cooling process, the momentum distribution develops a sharp peak near $q=0$ after very few iterations, as shown in Fig. 2(d).

The energy spread of the momentum distribution can be interpreted as a temperature, defined by $k_{B} T=2 J_{0}(\Delta q d)^{2}$, where for single particles $\Delta q$ is the half-width of the momentum distribution at $e^{-1 / 2}$ of the maximum value [13]. This is plotted in Fig. 2(e), and we find excellent agreement between our numerical calculations and analytical results obtained with Lévy statistics $[11,13]$. The latter predicts a final temperature $T \propto t^{-1}$ for square pulses, as shown in Fig. 2(e). For a zero-temperature reservoir, and parameters as in the caption of Fig. 2, we reach temperatures $T /\left(4 J^{0}\right) \sim 2 \times 10^{-3}$ in time $t_{f} J^{0} \sim 50$.

Finite reservoir temperature $T_{b}>0$ can lead to sympathetic heating of lattice atoms $a$ by absorption of thermal phonons, as described by $\hat{H}_{\text {int }}$. However, this process is forbidden by energy and momentum conservation, provided $J^{0}<\sqrt{\mu \omega_{R} m_{a} /\left(2 m_{b}\right)} / \pi$. In detail, energy conservation requires $c|\mathbf{k}|=\epsilon_{q}^{0}-\epsilon_{q^{\prime}}^{0}$ and conservation of momentum along the lattice direction leads to $k=q-$ $q^{\prime}(|k| \leq|\mathbf{k}|)$, where the atom $a$ is scattered from quasimomentum $q \approx 0 \rightarrow q^{\prime}$ by absorption of a phonon with momentum $\mathbf{k}$, and $c$ is the sound velocity in the BEC. These conditions cannot be fulfilled unless the above inequality is violated. Higher order processes involving two or more thermal phonons will be small. While in the above protocol we have switched off the decay during application of $\hat{E}_{j}$, we can leave decay switched on, provided that $\Gamma \ll$ $1 / \tau \ll\left|J^{1}\right|$. This will restrict the length of the possible pulses, thus slowing the cooling process.

The cooling scheme can be readily adapted to many bosons or fermions. For bosons, we assume that the collisional interaction between atoms $a$ is tuned to zero $\left(\hat{H}_{I} \rightarrow\right.$ $0)$. We work out the efficiency of the cooling protocol by deriving a quantum Boltzmann master equation (QBME) [18], which describes transitions between classical configurations of atoms occupying momentum states in the Bloch bands, $\mathbf{m}=\left[\left\{m_{q}^{0}\right\}_{q},\left\{m_{q}^{1}\right\}_{q}\right]$, where $m_{q}^{\alpha}$ is the occupation number of quasimomentum state $q$ in band $\alpha$. This is derived from the master equation by projection of the density operator $\rho$ onto diagonal elements, $\mathcal{P} \hat{\rho} \mathcal{P}=$ $\sum_{\mathbf{m}} w_{\mathbf{m}}|\mathbf{m}\rangle\langle\mathbf{m}|$, neglecting off-diagonal coherences. For the excitation step $\hat{E}_{j}$, the evolution is computed exactly from the excitation probability $P_{j}(q)$, and for the decay $\hat{D}$ we obtain

$$
\dot{w}_{\mathbf{m}}=\sum_{k, q} \Gamma_{k}\left[m_{q-k}^{0}\left(1 \pm m_{q}^{1}\right) w_{\mathbf{m}^{\prime}}-m_{q}^{1}\left(1 \pm m_{q-k}^{0}\right) w_{\mathbf{m}}\right],
$$

where $\mathbf{m}^{\prime}=\mathbf{m}-\mathbf{e}_{q-k, q}$ is the resulting configuration when a particle with quasimomentum $q$ in the upper band decays to the lower band with new quasimomentum $q-k$; i.e., $\mathbf{e}_{q-k, q}$ is a configuration vector with $m_{q-k}^{0}=1$, $m_{q}^{1}=-1$ and all other entries zero. The upper (lower) signs are for bosons (fermions). The approximation inherent in neglecting off-diagonal coherences plays a role only during the decay step, where these coherences couple to the occupation probabilities. We remark that an exact physical realization of the QBME can be obtained by modulating the lattice depth after each excitation step, randomizing the off-diagonal elements [19].

Figure 3(a) shows the decrease in temperature as a function of time for bosons and fermions, obtained from Monte Carlo simulations of the QBME [18]. For bosons, we use the same excitation pulse sequence as for a single atom in Fig. 2. The cooling process in this case outperforms that for a single atom, reaching low temperatures on shorter times due to bosonic enhancement (here the momentum width is found using a Gaussian fit). For fermions, the pulse sequence must be changed to create a dark-state region of quasimomenta with $|q|<q_{F}$, where $q_{F}$ is the Fermi momentum, in order to cool towards a $T=0$ Fermi distribution. In this case, time-square pulses are no longer efficient as there is a large secondary peak in $P_{j}(q)$ [see Fig. 2(b)], and we instead use Blackman pulses [11], which approach $P_{j}(q)=0$ monotonically. The momentum distribution develops the expected shape of a cold Fermi distribution after very few iterations [Fig. 3(b)] (we compute 


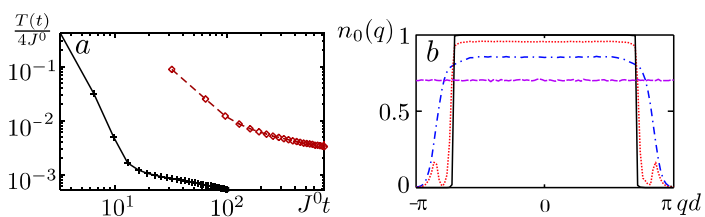

FIG. 3 (color online). Numerical simulation of the QBME: (a) Temperature as a function of cooling time for bosons (crosses) and fermions (circles). (b) Momentum distribution in band $\alpha=0$ for fermions after 0 (dashed line), 1 (dotted-dashed line), 2 (dotted line), and 20 (solid line) cooling cycles, each with $N_{p}=4$ pulses. Parameters used: Bosons: As for Figs. 2(a)-2(c), but $N=51$ particles. Fermions: $N=71, M=101$, Blackman pulses with $\quad \tau J^{0}=(1.78,1.78,14.2,14.2), \quad \delta q d=(0.19$, $-0.19,0.75,-0.75),(\omega-\delta)=(28.4,28.4,27.9,27.9) J^{0} ; V_{0}=$ $10 \omega_{R}, \omega_{R}=2 \pi \times 6 \mathrm{kHz}, m_{a} / m_{b}=1.74$, and $\Gamma=52.6 J^{0}$.

temperatures from these results by fitting a Fermi distribution function).

Our model predicts that the temperature will always decrease with increasing cooling time. Experimental imperfections will, in practice, give rise to decoherence and heating (e.g., from spontaneous emissions [2] or scattering multiple phonons). One assumption made above for bosons was that the interaction between lattice atoms $a$ is approximately zero. Using time-dependent density matrix renormalization group (DMRG) methods [20], we computed the population remaining in the lowest band after an excitation pulse with interactions present [21]. For $U^{\alpha \alpha^{\prime}} \ll 1 / \tau \ll$ $\left|J^{1}\right|$, there is no significant change in the excitation profile, and, to ensure that the ground state is not substantially altered, we require $U^{\alpha, \alpha^{\prime}} \ll J^{0}$, under which conditions the above conclusions should not change. Another assumption is that we have a homogeneous background potential, which is a requirement for the realization of many strongly correlated phases independent of the cooling techniques employed. In this context, the final temperatures will be limited as the cooled atoms become localized around inhomogeneities. In practice, development of these techniques should be iterative, as advances in clean flatbottomed traps will allow better cooling, and colder temperatures will increase sensitivity of the atoms to the remaining imperfections.

Finally, after the cooling, we adiabatically ramp up the interaction strength to produce an interacting system, assuming that the system is decoupled from the reservoir $b$. Using time-dependent DMRG methods [20], we have computed the evolution when ramping from a noninteracting to a hard-core Bose lattice gas in 1D (Tonks gas) in the Bose-Hubbard model $\left(H_{a}\right.$ with no Raman coupling and only the lowest band). For ramping times on the order of $10 / J^{0}$, we observe negligible heating [21].

In a more general context, we can see these formal analogies between cold atoms in optical lattices and familiar quantum optical systems, their coherent control and dissipative dynamics, as just one example to realize and apply quantum optical concepts and techniques in a new setting.
We thank P. Rabl for discussions. This work was supported by OLAQUI. Work in Innsbruck was supported by Austrian Science Foundation, SCALA, and IQI, and work in Oxford by EPSRC Project No. EP/C51933/1.

[1] L. Pitaevskii and S. Stringari, Bose-Einstein Condensation (Oxford University Press, Oxford, 2003).

[2] D. Jaksch and P. Zoller, Ann. Phys. (N.Y.) 315, 52 (2005); M. Lewenstein et al., cond-mat/0606771.

[3] K. Winkler et al., Nature (London) 441, 853 (2006); S. Fölling et al., cond-mat/0606592; G. K. Campbell et al., cond-mat/0606642; T. Volz et al., Nature Phys. 2, 692 (2006); J. Sebby-Strabley et al., cond-mat/0602103; K. Günter et al., Phys. Rev. Lett. 96, 180402 (2006); S. Ospelkaus et al., ibid. 96, 180403 (2006); L. Fallani et al., cond-mat/0603655.

[4] A. Micheli, G. K. Brennen, and P. Zoller, Nature Phys. 2, 341 (2006); L. Santos et al., Phys. Rev. Lett. 93, 030601 (2004); J. J. Garcia-Ripoll, M. A. Martin-Delgado, and J. I. Cirac, ibid. 93, 250405 (2004).

[5] S. Trebst, U. Schollwöck, M. Troyer, and P. Zoller, Phys. Rev. Lett. 96, 250402 (2006).

[6] A. J. Daley, P. O. Fedichev, and P. Zoller, Phys. Rev. A 69, 022306 (2004).

[7] A. Griessner, A. J. Daley, D. Jaksch, and P. Zoller, Phys. Rev. A 72, 032332 (2005).

[8] This is in contrast to filtering schemes, which work above unit filling and rely on a gap provided by atomic on site interactions to achieve exactly one atom per site $[9,10]$.

[9] P. Rabl et al., Phys. Rev. Lett. 91, 110403 (2003).

[10] M. Popp, J. J. Garcia-Ripoll, K. G. H. Vollbrecht, and J. I. Cirac, Phys. Rev. A 74, 013622 (2006).

[11] M. Kasevich and S. Chu, Phys. Rev. Lett. 69, 1741 (1992); J. Reichel et al., ibid. 75, 4575 (1995).

[12] H. J. Metcalf and P. van der Straten, Laser Cooling and Trapping (Springer-Verlag, New York, 1999).

[13] F. Bardou, J.P. Bouchaud, A. Aspect, and C. CohenTannoudji, Lévy Statistics and Laser Cooling (Cambridge University Press, Cambridge, England, 2002).

[14] E. A. Donley, N. R. Claussen, S. T. Thompson, and C.E. Wieman, Nature (London) 417, 529 (2002); T. Loftus et al., Phys. Rev. Lett. 88, 173201 (2002); M. Theis et al., ibid. 93, 123001 (2004).

[15] We neglect reabsorption as discussed in laser cooling [16]. These effects can be suppressed, e.g., by evaporative cooling of the reservoir [7].

[16] Y. Castin, J. I. Cirac, and M. Lewenstein, Phys. Rev. Lett. 80, 5305 (1998).

[17] See C.W. Gardiner and P. Zoller, Quantum Noise (Springer, Berlin, 2005), 3rd ed., and references therein.

[18] C. W. Gardiner and P. Zoller, Phys. Rev. A 55, 2902 (1997); D. Jaksch, C. W. Gardiner, and P. Zoller, Phys. Rev. A 56, 575 (1997).

[19] This is reminiscent of the twirl in state purification protocols: D. Deutsch et al., Phys. Rev. Lett. 77, 2818 (1996).

[20] G. Vidal, Phys. Rev. Lett. 91, 147902 (2003); A. J. Daley et al., J. Stat. Mech. (2004) P04005; S. R. White and A. E. Feiguin, Phys. Rev. Lett. 93, 076401 (2004); F. Verstraete, J. J. Garcia-Ripoll, and J. I. Cirac, ibid. 93, 207204 (2004).

[21] A. Griessner et al. (to be published). 